\title{
Niemeier Lattices in the Free Fermionic Heterotic-String Formulation
}

\author{
Panos Athanasopoulos and Alon E. Faraggi \\ Department of Mathematical Sciences, University of Liverpool, Liverpool L69 7ZL, UK \\ Correspondence should be addressed to Alon E. Faraggi; alon.faraggi@liv.ac.uk
}

Received 9 May 2017; Accepted 13 June 2017; Published 10 August 2017

Academic Editor: Dimitrios Tsimpis

Copyright (c) 2017 Panos Athanasopoulos and Alon E. Faraggi. This is an open access article distributed under the Creative Commons Attribution License, which permits unrestricted use, distribution, and reproduction in any medium, provided the original work is properly cited.

\begin{abstract}
The spinor-vector duality was discovered in free fermionic constructions of the heterotic string in four dimensions. It played a key role in the construction of heterotic-string models with an anomaly-free extra $Z^{\prime}$ symmetry that may remain unbroken down to low energy scales. A generic signature of the low scale string derived $Z^{\prime}$ model is via diphoton excess that may be within reach of the LHC. A fascinating possibility is that the spinor-vector duality symmetry is rooted in the structure of the heterotic-string compactifications to two dimensions. The two-dimensional heterotic-string theories are in turn related to the so-called moonshine symmetries that underlie the two-dimensional compactifications. In this paper, we embark on exploration of this connection by the free fermionic formulation to classify the symmetries of the two-dimensional heterotic-string theories. We use two complementary approaches in our classification. The first utilises a construction which is akin to the one used in the spinor-vector duality. Underlying this method is the triality property of $S O(8)$ representations. In the second approach, we use the free fermionic tools to classify the twenty-four-dimensional Niemeier lattices.
\end{abstract}

\section{Introduction}

The ATLAS and CMS collaborations reported in December 2015 evidence for excess in the diphoton channel [1, 2]. Absence of evidence for any other deviation from the Standard Model expected signals suggested that the excess could be interpreted as production and decay of a Standard Model singlet state by heavy vector-like states [3], in a process depicted in Figure 1. In [4], it was shown that the spectrum required to generate the excess naturally arises in the string derived model of [5], which allows for a light $Z^{\prime}$ vector boson. Anomaly cancellation mandates that the mass scale of the Standard Model singlet state, which is produced in resonance in Figure 1, as well as the mass scale of the heavy vector-like states that are used in the production and decay of the singlet states, be the $Z^{\prime}$ symmetry breaking scale. Thus, assuming that the $Z^{\prime}$ remains unbroken down to the multi$\mathrm{TeV}$ scale naturally gives rise to the characteristics required to generate diphoton excess. In [6], it was shown that existence of the light $Z^{\prime}$ at the multi-TeV scale is compatible with gauge coupling unification at the GUT scale, as well as other phenomenological constraints.

In August 2016, the ATLAS and CMS collaborations reported that accumulation of further data did not substantiate the observation of the diphoton excess $[7,8]$, suggesting that initial observation was a statistical fluctuation. However, this does not repudiate the diphoton excess as a signal of the string derived $Z^{\prime}$ model, albeit not as the purported $750 \mathrm{GeV}$ resonance. Thus, searching for diphoton excesses in the energy range accessible at the LHC continues to be of immense interest.

Extra $Z^{\prime}$ vector bosons as possible signatures of heterotic-string vacua have been discussed in the literature since the mid-eighties [9-11]. The difficulty in constructing heterotic-string models that allow for an extra $U(1)$ symmetry to remain unbroken down to low scales stems from the fact that the aforementioned symmetries tend to be anomalous in the heterotic-string derived models. The reason is that the string models utilise the symmetry breaking pattern $E_{6} \rightarrow S O(10) \times U(1)_{\zeta}$, with anomalous 


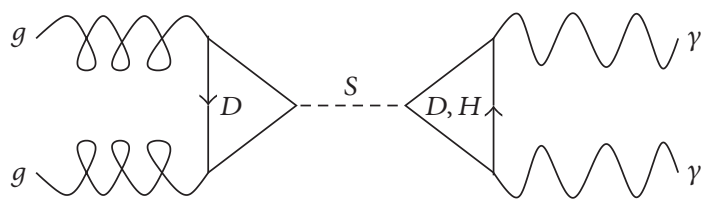

Figure 1: Production and diphoton decay of the Standard Model singlet scalar state.

$U(1)_{\zeta}$. Suppression of left-handed neutrino masses implies that the extra $U(1)$ symmetry, which is embedded in $S O(10)$, has to be broken near the GUT scale. This conundrum motivated the search of extra $U(1)$ symmetries that do not admit the $E_{6}$ embedding of their charges $[12,13]$. However, these choices result in contradiction between gauge coupling unification and the gauge coupling parameters at the electroweak scale, which works well if the extra $U(1)$ charges admit the $E_{6}$ embedding $[6,14,15]$.

It is therefore notable that in [5] a heterotic-string derived model with an anomaly-free $U(1)_{\zeta}$ was constructed. What is perhaps more remarkable is that the construction of the model utilises a basic duality symmetry that operates in the space of $Z_{2} \times Z_{2}$ heterotic-string vacua which was dubbed spinor-vector duality. The duality operates under the exchange of the total number of spinorial $16 \oplus \overline{16}$ and vectorial 10 representations of $S O(10)$. For every vacuum with a number of $16 \oplus \overline{16}$ representations and a number of 10 representations, there exists a dual vacuum in which the two numbers are interchanged. One can further show that the duality arises from the breaking of $(2,2)$ world-sheet supersymmetry to $(2,0)$ and that the duality map is induced by a spectral flow operator that operates in the bosonic sector of the heterotic-string vacuum. In the vacua with $(2,2)$ world-sheet supersymmetry, the $S O(10) \times U(1)_{\zeta}$ symmetry is enhanced to $E_{6}$. The chiral 27 and $\overline{27}$ representations of $E_{6}$ decompose under $S O(10) \times U(1)_{\zeta}$ as

$$
\begin{aligned}
& 27=16_{+1 / 2}+10_{-1}+1_{+2}, \\
& \overline{27}=\overline{16}_{-1 / 2}+10_{+1}+1_{-2} .
\end{aligned}
$$

Thus, the $(2,2)$ vacua are self-dual under the exchange of the total number of spinorial $16 \oplus \overline{16}$ and vectorial 10 representations. The spectral flow operator acts as the $U(1)$ generator of the $N=2$ world-sheet supersymmetry and interchanges between the $S O(10)$ components in the decomposition of $E_{6}$ under $S O(10) \times U(1)_{\zeta}$. The breaking of $E_{6}$ to $S O(10) \times$ $U(1)_{\zeta}$ or the breaking of the world-sheet supersymmetry from $(2,2)$ to $(2,0)$ is induced by Wilson lines. One choice of Wilson line breaking results in a vacuum with $\#_{1}$ of $16 \oplus$ $\overline{16}$ representations and $\#_{2}$ of 10 representations, whereas a second choice interchanges the two numbers. Furthermore, the duality map between the dual cases is induced by the spectral flow operator of the parent $(2,2)$ vacuum.

A new twist is that the spinor-vector duality was used to construct the heterotic-string model with anomaly-free $U(1)_{\zeta}$ that allows for an extra $E_{6} Z^{\prime}$ to remain unbroken down to low scales. Using the methods developed in [1623] for the classification of free fermionic models, a selfdual model under the spinor-vector duality is fished from the landscape of vacua. The unbroken gauge symmetry at the string level is $S O(10) \times U(1)_{\zeta}$, but the spectrum is selfdual under the exchange of the total number of spinorial $16 \oplus \overline{16}$ and vectorial 10 representations. Thus, the spectrum still forms complete $E_{6}$ multiplets and consequently $U(1)_{\zeta}$ is anomaly-free. This is possible in the $Z_{2} \times Z_{2}$ orbifold if the different spinorial and vectorial components are obtained from different fixed points. Conversely, obtaining both the spinorial and the vectorial representations at the same fixed point necessarily implies that the gauge symmetry is enhanced to $E_{6}$. In the model of [5], the $S O(10)$ symmetry is broken at the string level to $S O(6) \times S O(4)$. However, the chiral spectrum of the model still appears in complete $E_{6}$ representations, hence maintaining $U(1)_{\zeta}$ as an anomaly-free symmetry.

The spinor-vector duality is a fundamental symmetry in the space of $(2,0)$ heterotic-string vacua. It played a central role in the construction of the $Z^{\prime}$ model in [5]. If the additional $U(1)$ symmetry remains unbroken down to low scales, it may be detected via diphoton production as in Figure 1.

Another fascinating direction of investigation is the possibility that the spinor-vector duality is a mere reflection of a much larger symmetry structure that underlies this class of vacua. The much larger symmetry structure is obtained in compactifications to two dimensions and gives rise to 24dimensional lattices. Reference [24] alluded to possible similarities with the Massive Spectrum boson-fermion Degeneracy Symmetry (MSDS) $[25,26]$, which arises from a basic Jacobi-like identity in 24 dimensions. The compactifications to two dimensions are connected to 24-dimensional lattices and the symmetries of those are related to the so-called moonshine symmetries. In two dimensions, the spectral flow operator that induces the spinor-vector duality and the twist operators which acts on the internal coordinates can be seen to share a common structure in that both have four periodic right-moving fermions. One may further envision that, under decompactification back to 4 dimensions, the two spinor-vector dual vacua appear on the boundaries of the moduli space. This is reminiscent of the case when space-time supersymmetry is broken to $N=0$ by a Scherk-Schwarz mechanism in nine dimensions and the supersymmetric and nonsupersymmetric vacua appear on the boundaries of the compactified dimension.

In this paper, we embark on a program to explore the connection between the moonshine symmetries and the spinor-vector duality. We foresee that the spinor-vector duality is a tip of the iceberg and that elucidation of this connection may reveal a covering space of large space of string compactifications facilitating a deeper understanding of their symmetries and connections. In this paper, we make several modest steps to initiate the enterprise. In Section 2, we review a specific realisation of the spinor-vector duality, which is particularly suited for our purpose here. In this realisation, the untwisted vector bosons corresponding to the sixteen-dimensional vector bundle of the heterotic string in 
ten dimensions generate an $\mathrm{SO}(8)^{4}$ gauge symmetry. This is obtained by including in the construction four basis vectors with four periodic world-sheet fermions, and enhancement to larger gauge symmetries is obtained from twisted sectors. A similar basis vector with four periodic fermions that acts simultaneously on the gauge degrees of freedom and the internal coordinates produces the twisted sectors. The spinor-vector duality can be then seen to arise due to a special choice of the Generalised GSO (GGSO) phases. In Section 3, we explore a similar construction in two dimensions and classify the symmetries that arise on the resulting 24-dimensional lattices. In Section 4, we derive representations of some of the Niemeier lattices in 24 dimensions in the free fermionic formulation. Section 5 concludes our paper.

\section{A Novel Basis}

In this section, we review the spinor-vector duality in the specific realisation of [27]. Construction of a consistent four-dimensional heterotic-string theory in the light-cone gauge requires 20 left-moving and 44 right-moving twodimensional real fermions [28, 29] propagating on the world-sheet torus. The models in this construction are specified in terms of a set of basis vectors $v_{i}, i=1, \ldots, n$,

$$
v_{i}=\left\{\alpha_{i}\left(f_{1}\right), \alpha_{i}\left(f_{2}\right), \alpha_{i}\left(f_{3}\right) \cdots\right\}
$$

describing the transformation properties of each fermion

$$
f_{A} \longrightarrow-e^{i \pi \alpha_{i}\left(f_{A}\right)} f_{A}, \quad A=1, \ldots, 44,
$$

when transported along the noncontractible loops of the oneloop vacuum to vacuum amplitude. The basis vectors span a space $\Xi$ which consists of $2^{N}$ sectors that give rise to the string spectrum. Each sector is given by

$$
\xi=\sum N_{i} v_{i}, \quad N_{i}=0,1 .
$$

The spectrum is truncated by a GGSO projection whose action on a string state $|S\rangle$ is

$$
e^{i \pi v_{i} \cdot F_{S}}|S\rangle=\delta_{S} c\left[\begin{array}{l}
S \\
v_{i}
\end{array}\right]|S\rangle,
$$

where $F_{S}$ is the fermion number operator and $\delta_{S}= \pm 1$ is the space-time spin statistics index. Different sets of projection coefficients $c\left[\begin{array}{c}S \\ v_{i}\end{array}\right]= \pm 1$ consistent with modular invariance give rise to different models. A model is defined by a set of basis vectors $v_{i}, i=1, \ldots, n$, and a set of $2^{N(N-1) / 2}$ independent projection coefficients $c\left[\begin{array}{l}v_{i} \\ v_{j}\end{array}\right], i>j$. The 64 world-sheet fermions in the light-cone gauge are denoted by $\psi^{\mu}, \chi^{i}, y^{i}, \omega^{i}, i=1, \ldots, 6$ (real left-moving fermions) and $\bar{y}^{i}, \bar{\omega}^{i}, i=1, \ldots, 6$ (real right-moving fermions); $\bar{\psi}^{j}, j=1, \ldots, 4 ; \bar{\eta}^{k}, k=0,1,2,3 ; \bar{\phi}^{l}, l=1, \ldots, 8$ (complex right-moving fermions). The division of the rightmoving complex fermions into groups of four is obtained by introducing four basis vectors $z_{\{0,1,2,3\}}$ into the basis. Each of $z_{i}$ contains four nonoverlapping periodic fermions under the sets $\left\{\bar{\psi}^{1, \ldots, 4}, \bar{\eta}^{0,1,2,3}, \bar{\phi}^{1, \ldots, 4}, \bar{\phi}^{5, \ldots, 8}\right\}$. We note that our notation here deviates from the conventional one in the free fermion literature by renaming $\bar{\psi}^{5} \equiv \bar{\eta}^{0}$. To illustrate the structure of the spinor-vector duality, we use a basis $V$ of seven boundary condition basis vectors given by

$$
V=\left\{v_{1}, v_{2}, \ldots, v_{7}\right\}
$$

where

$$
\begin{aligned}
& v_{1}=\mathbf{1}=\left\{\psi^{\mu}, \chi^{1, \ldots, 6}, y^{1, \ldots, 6}, \omega^{1, \ldots, 6} \mid \bar{y}^{1, \ldots, 6}, \bar{\omega}^{1, \ldots, 6}, \bar{\eta}^{1,2,3},\right. \\
& \left.\bar{\psi}^{1, \ldots, 5}, \bar{\phi}^{1, \ldots, 8}\right\}, \\
& v_{2}=S=\left\{\psi^{\mu}, \chi^{1, \ldots, 6}\right\}, \\
& v_{3}=z_{1}=\left\{\bar{\phi}^{1, \ldots, 4}\right\}, \\
& v_{4}=z_{2}=\left\{\bar{\phi}^{5, \ldots, 8}\right\}, \\
& v_{5}=z_{3}=\left\{\bar{\psi}^{1, \ldots, 4}\right\}, \\
& v_{6}=z_{0}=\left\{\bar{\eta}^{0,1,2,3}\right\}, \\
& v_{7}=b_{1}=\left\{\chi^{34}, \chi^{56}, y^{34}, y^{56} \mid \bar{y}^{34}, \bar{y}^{56}, \bar{\eta}^{0}, \bar{\eta}^{1}\right\} .
\end{aligned}
$$

The partition function of such models is of the form

$$
\begin{aligned}
& Z(\tau, \bar{\tau})=\frac{1}{\tau_{2}(\eta \bar{\eta})^{2}} \frac{1}{\eta^{10} \bar{\eta}^{22}} \frac{1}{2^{7}} \sum_{a, b, s, s^{\prime}} \sum_{h_{1}, g_{1}} \sum_{H_{I}, G_{I}} C \\
& \cdot\left[\begin{array}{c}
a, s, h_{1}, H_{I} \\
b, s^{\prime}, g_{1}, G_{I}
\end{array}\right] \theta\left[\begin{array}{l}
a+s \\
b+s^{\prime}
\end{array}\right] \theta\left[\begin{array}{l}
a+s \\
b+s^{\prime}
\end{array}\right] \theta \\
& \cdot\left[\begin{array}{l}
a+s+h_{1} \\
b+s^{\prime}+g_{1}
\end{array}\right] \theta\left[\begin{array}{l}
a+s \\
b+s^{\prime}
\end{array}\right] \theta\left[\begin{array}{l}
a \\
b
\end{array}\right] \theta \\
& \cdot\left[\begin{array}{l}
a+h_{1} \\
b+g_{1}
\end{array}\right] \theta\left[\begin{array}{l}
a+h_{1} \\
b+g_{1}
\end{array}\right] \theta\left[\begin{array}{l}
a \\
b
\end{array}\right] \theta \\
& \cdot\left[\begin{array}{l}
a+h_{1} \\
b+g_{1}
\end{array}\right] \theta\left[\begin{array}{l}
a+h_{1} \\
b+g_{1}
\end{array}\right] \bar{\theta}\left[\begin{array}{l}
a \\
b
\end{array}\right] \bar{\theta} \\
& \cdot\left[\begin{array}{l}
a+h_{1} \\
b+g_{1}
\end{array}\right] \bar{\theta}\left[\begin{array}{l}
a+h_{1} \\
b+g_{1}
\end{array}\right] \bar{\theta}\left[\begin{array}{l}
a \\
b
\end{array}\right] \bar{\theta} \\
& \cdot\left[\begin{array}{l}
a+h_{1} \\
b+g_{1}
\end{array}\right] \bar{\theta}\left[\begin{array}{l}
a+h_{1} \\
b+g_{1}
\end{array}\right] \bar{\theta}\left[\begin{array}{l}
a+H_{0} \\
b+G_{0}
\end{array}\right] \bar{\theta} \\
& \cdot\left[\begin{array}{c}
a+H_{0} \\
b+G_{0}
\end{array}\right] \bar{\theta}\left[\begin{array}{l}
a+H_{0} \\
b+G_{0}
\end{array}\right] \bar{\theta} \\
& \cdot\left[\begin{array}{l}
a+H_{0} \\
b+G_{0}
\end{array}\right] \bar{\theta}\left[\begin{array}{l}
a+H_{3} \\
b+G_{3}
\end{array}\right] \bar{\theta}\left[\begin{array}{l}
a+H_{3} \\
b+G_{3}
\end{array}\right] \bar{\theta} \\
& \cdot\left[\begin{array}{c}
a+H_{3} \\
b+G_{3}
\end{array}\right] \bar{\theta}\left[\begin{array}{l}
a+H_{3} \\
b+G_{3}
\end{array}\right] \bar{\theta}\left[\begin{array}{c}
a+H_{1} \\
b+G_{1}
\end{array}\right] \bar{\theta}
\end{aligned}
$$




$$
\begin{aligned}
& \cdot\left[\begin{array}{l}
a+H_{1} \\
b+G_{1}
\end{array}\right] \bar{\theta}\left[\begin{array}{l}
a+H_{1} \\
b+G_{1}
\end{array}\right] \bar{\theta}\left[\begin{array}{l}
a+H_{1} \\
b+G_{1}
\end{array}\right] \bar{\theta} \\
& \cdot\left[\begin{array}{l}
a+H_{2} \\
b+G_{2}
\end{array}\right] \bar{\theta}\left[\begin{array}{l}
a+H_{2} \\
b+G_{2}
\end{array}\right] \bar{\theta} \\
& \cdot\left[\begin{array}{l}
a+H_{2} \\
b+G_{2}
\end{array}\right] \bar{\theta}\left[\begin{array}{l}
a+H_{2} \\
b+G_{2}
\end{array}\right] .
\end{aligned}
$$

The phases $C\left[\begin{array}{c}a, s, h_{1}, H_{I} \\ b, s^{\prime}, g_{1}, G_{I}\end{array}\right]$ can be calculated in terms of the phases $C\left[\begin{array}{l}v_{i} \\ v_{j}\end{array}\right]$ that define the model: if we define the vectors

$$
\begin{gathered}
\alpha=a \mathbf{1}+s S+h_{1} b_{1}+\sum_{I} H_{I} z_{I}=\sum n_{a} v_{a}, \\
\alpha^{\prime}=b \mathbf{1}+s^{\prime} S+g_{1} b_{1}+\sum_{I} G_{I} z_{I}=\sum n_{b}^{\prime} v_{b},
\end{gathered}
$$

then

$$
\begin{aligned}
& C\left[\begin{array}{l}
a, s, h_{1}, H_{I} \\
b, s^{\prime}, g_{1}, G_{I}
\end{array}\right]=C\left[\begin{array}{l}
\alpha \\
\alpha^{\prime}
\end{array}\right] \\
& \quad=\left(\delta_{\alpha}\right)^{\sum_{a} n_{a}^{\prime}-1}\left(\delta_{\alpha^{\prime}}\right)^{\sum_{a} n_{a}-1} e^{-\pi i r(\alpha) \cdot \alpha^{\prime}} \prod_{a, b} C\left[\begin{array}{l}
\mathbf{B}_{a} \\
\mathbf{B}_{b}
\end{array}\right]^{n_{a} n_{b}^{\prime}}
\end{aligned}
$$

where $\delta_{\alpha}=e^{i \pi \alpha\left(\psi^{\mu}\right)}$ and $r(\alpha)=(\alpha-[\alpha]) / 2$ is the reduction vector which takes $\alpha$ to $[\alpha]$ with the latter having all its entries in the interval $(-1,1]$.

The models generated by basis (7) preserve $N=2$ space-time supersymmetry. Models that break $N=2$ to $N=1$ space-time supersymmetry are easily incorporated by introducing a second basis vector $b_{2}[18,19]$. The second function of the second $Z_{2}$ basis vector $b_{2}$ is to break the untwisted observable symmetry gauge group from $S O(12) \times$ $S O(4)$ to $S O(10) \times U(1)^{3}$. Here, the spinor-vector duality is therefore seen in terms of $S O(12)$, rather than $S O(10)$, representations. However, since in the $N=1$ vacua the spinor-vector duality operates separately on each of the $N=2$ planes $[18,19]$, the discussion in terms of $N=2$ representations is sufficient.

In the models generated by the basis in (7), all the geometrical degrees of freedom $\left\{y^{i}, \omega^{i} \mid \bar{y}^{i}, \bar{\omega}^{i}\right\}, i=1, \ldots, 3$, are grouped together. The remaining 8 left-moving and 32 right-moving world-sheet fermions are divided into five nonoverlapping groups of eight real fermions. In the tendimensional supersymmetric heterotic string, such a division always produces either $S O(32)$ or $E_{8} \times E_{8}$ gauge groups [30]. Although naively one may expect that other gauge symmetries, such as $S O(8)^{4}, S O(16)^{2}$, or $S O(8) \times S O(24)$, may be obtained, the modular properties of the partition function forbid the other possible extensions. In terms of the $S O(8)$ characters, this property follows from the equivalence of the $8_{V}, 8_{S}$, and $8_{C} S O(8)$ representations, which enables twisted constructions of the $S O(32)$ or $E_{8} \times E_{8}$ gauge groups. These phenomena appear in the models generated by the basis in
(7) and will be exploited in Section 3. The basis vector $b_{1}$ generates a $Z_{2}$ projection which breaks $N=4$ to $N=$ 2 space-time supersymmetry and breaks one of the $S O(8)$ groups to $S O(4) \times S O(4) \equiv S U(2)^{4}$.

The sectors contributing to the gauge group are the 0 sector and the 10 antiholomorphic sets:

$$
\begin{aligned}
& G \\
& =\left\{\begin{array}{c}
0, \\
z_{0}, z_{1}, z_{2}, z_{3}, \\
z_{0}+z_{1}, z_{0}+z_{2}, z_{0}+z_{3}, z_{1}+z_{2}, z_{1}+z_{3}, z_{2}+z_{3}
\end{array}\right\} .
\end{aligned}
$$

The 0 sector requires two oscillators acting on the vacuum in the right-moving sector to produce a massless state; the $z_{j}$ sectors require one oscillator; and the $z_{i}+z_{j}$ sectors require no oscillators. We first discuss the $N=4$ gauge group arising prior to the inclusion of the basis vector $b_{1}$, which reduces $N=4$ to $N=2$ space-time supersymmetry. The basis vector $b_{1}$ does not produce additional enhancement sectors and therefore merely breaks the $N=4$ gauge group to a subgroup.

The 0 sector gauge bosons produce the gauge symmetry

$$
[S O(12)] \times S O(8)^{4},
$$

where the $S O(12)$ group factor arises from the 12 rightmoving world-sheet fermions $\{\bar{y}, \bar{\omega}\}^{1, \ldots, 6}$, which correspond to the internal lattice at the free fermionic $S O(12)$ enhanced symmetry point. The $S O(8)_{3,0,1,2}$ group factors arise, respectively, from $\bar{\psi}^{1, \ldots, 4}, \bar{\eta}^{0,1,2,3}, \bar{\phi}^{1, \ldots, 4}$, and $\bar{\phi}^{5, \ldots, 8}$. The notation adheres to the conventional notation in the quasi-realistic heterotic-string models in the free fermionic formulation [31-39].

The additional sectors in (11) may produce space-time vector bosons that enhance the untwisted four-dimensional gauge symmetry. The possible enhancements depend on the GGSO projection coefficients $c\left[\begin{array}{l}z_{i} \\ z_{j}\end{array}\right]$ with $i \neq j$. Excluding the basis vector $b_{1}$, all vacua possess $N=4$ space-time supersymmetry, which fixes the $c\left[\begin{array}{c}S \\ z_{i}\end{array}\right]$ phases. Hence, there may be a priori $2^{6}$ possibilities for the four-dimensional gauge group, some of which are repeated. Identical manifestations of the gauge groups arise from twisted realisation of the group generators, due to the triality property of the $S O(8)$ group representations. This is the four-dimensional manifestation of the twisted generation of gauge groups already observed in the ten-dimensional case. A few of the possibilities that may arise were classified in [27]. The same construction will be exploited in Section 3 in the analysis of compactifications to two dimensions.

2.1. A Simple Example of the Spinor-Vector Duality. The basis vector $b_{1}$ reduces $N=4 \rightarrow N=2$ space-time supersymmetry. The $N=4$ vacuum with $[S O(12)] \times S O(16) \times$ 
$S O(16)$ gauge group is realised with the GGSO projection coefficient taken to be

$$
\begin{aligned}
c\left[\begin{array}{l}
z_{0} \\
z_{1}
\end{array}\right] & =c\left[\begin{array}{l}
z_{0} \\
z_{3}
\end{array}\right]=c\left[\begin{array}{l}
z_{1} \\
z_{2}
\end{array}\right]=-c\left[\begin{array}{l}
z_{0} \\
z_{2}
\end{array}\right]=-c\left[\begin{array}{l}
z_{1} \\
z_{3}
\end{array}\right] \\
& =-c\left[\begin{array}{l}
z_{2} \\
z_{3}
\end{array}\right]=-1 .
\end{aligned}
$$

With this set of GGSO phases, the additional sectors, beyond the 0 sector, that produce additional space-time vector bosons are $z_{2}$ and $z_{3}$, whereas those from all other sectors in (11) are projected out. The additional projection induced by the basis vector $b_{1}$ breaks the gauge symmetry arising from the 0 sector to

$$
\begin{aligned}
& {[\mathrm{SO}(8) \times \mathrm{SO}(4)]_{\mathscr{L}} \times\left[\mathrm{SO}(8)_{3} \times \mathrm{SO}(4) \times \mathrm{SO}(4)\right]_{\mathrm{O}}} \\
& \quad \times\left[\mathrm{SO}(8)_{1} \times \mathrm{SO}(8)_{2}\right]_{H} .
\end{aligned}
$$

The $S O(12)$ lattice gauge symmetry in (12) is reduced to $[S O(8) \times S O(4)]_{\mathscr{L}}$. The observable gauge symmetry arising from the 0 sector is $\left[S O(8)_{3} \times S O(4) \times S O(4)\right]_{O}$, and $\left[S O(8)_{1} \times\right.$ $\left.\mathrm{SO}(8)_{2}\right]_{H}$ is the hidden gauge symmetry. Both observable and hidden sector gauge symmetries are enhanced. The hidden gauge symmetry is enhanced to $[S O(16)]_{H}$ by the additional vector bosons arising from the sector $z_{2}$. At the $N=4$ level, the additional vector bosons from the sector $z_{3}$ enhance the observable $\left[\mathrm{SO}(8)_{3} \times \mathrm{SO}(8)_{0}\right]_{O}$ gauge symmetry to $[\mathrm{SO}(16)]_{O}$. At the $N=2$ level, the $b_{1}$ projection reduces $[S O(16)]_{O} \rightarrow$ $[S O(12) \times S O(4)]_{O} \equiv\left[S O(12) \times S U(2)_{0} \times S U(2)_{1}\right]_{O}$. The $N=2$ spinor-vector duality is realised by the exchange of the vectorial 12 representation of $S O(12)$ with the spinorial 32 representation. This duality is illustrated by considering two different models in which these representations are interchanged due to the choices of the GGSO projection coefficients. We remark further that the choice of GGSO projection coefficients in (13) prevents the enhancement of the $S O(12) \times S U(2)$ gauge symmetry to $E_{7}$, which is the $N=2$ analog of the enhancement of $S O(10) \times U(1)$ to $E_{6}$ at the $N=1$ level.

The first choice of the extra GGSO projection coefficients that we consider is given by

$$
c\left[\begin{array}{c}
b_{1} \\
1, z_{0}
\end{array}\right]=-c\left[\begin{array}{c}
b_{1} \\
S, z_{1}, z_{2}, z_{3}
\end{array}\right]=-1 .
$$

This choice defines a model with 2 multiplets in $\left(1,2_{L}+\right.$ $\left.2_{R}, 12,1,2,1\right)$ and 2 in $\left(8,2_{L}+2_{R}, 1,2,1,1\right)$ representations of

$$
\begin{aligned}
& {[S O(8) \times S O(4)]_{\mathscr{L}} \times\left[S O(12) \times S U(2)_{0} \times S U(2)_{1}\right]_{O}} \\
& \quad \times[S O(16)]_{H} .
\end{aligned}
$$

The sectors producing the vectorial 12 representation of $S O(12)$ are the sectors $b_{1}$ and $b_{1}+z_{3}$, where the sector $b_{1}$ produces the $(1,2,2)$ representation and the sector $b_{1}+z_{3}$ produces $\left(8_{S}, 1,1\right)$ under the decomposition

$$
\begin{gathered}
{[\mathrm{SO}(12)]_{O} \longrightarrow} \\
{[\mathrm{SO}(8) \times \mathrm{SO}(4)]_{O} \equiv[S O(8) \times S U(2) \times S U(2)]_{O} .}
\end{gathered}
$$

All other states are projected out. In this case, there are eight multiplets in the vectorial representation of the observable $S O(12)$, which also transform as doublets of the observable $S U(2)_{1}$.

We next consider the choice of GGSO phases given by

$$
c\left[\begin{array}{c}
b_{1} \\
1, z_{0}, z_{1}
\end{array}\right]=-c\left[\begin{array}{c}
b_{1} \\
S, z_{2}, z_{3}
\end{array}\right]=-1 .
$$

This case defines a model with 2 multiplets in $\left(1,2_{L}+\right.$ $\left.2_{R}, 32,1,1,1\right)$ and 2 in $\left(1,2_{L}+2_{R}, 1,1,2,16\right)$ representations of the gauge group in (16). The sectors producing the spinorial 32 representation of $[S O(12)]_{O}$ are the sectors $b_{1}+z_{0}$ and $b_{1}+z_{3}+z_{0}$, where the sector $b_{1}+z_{0}$ produces the $\left(8_{V}, 2,1\right)$ representation and the sector $b_{1}+z_{3}+z_{0}$ produces $\left(8_{C}, 1,2\right)$ under the decomposition given in (17). The sectors producing the vectorial 16 multiplet of the hidden $S O(16)$ gauge group are the sectors $b_{1}$ and $b_{1}+z_{2}$, where the sector $b_{1}$ produces the $\left(8_{V}, 1\right)$ multiplet and the sector $b_{1}+z_{2}$ produces the $\left(1,8_{C}\right)$ multiplet under the decomposition $[\mathrm{SO}(16)]_{H} \rightarrow\left[\mathrm{SO}(8)_{1} \times\right.$ $\left.\mathrm{SO}(8)_{2}\right]_{H}$. The hidden 16 multiplets transform as doublets of the observable $S U(2)_{1}$ group. All other states are projected out. In this model, there are eight multiplets in the spinorial 32 representation of the observable $[S O(12)]_{O}$.

We note that, in the first model, the vectorial 12 representation of the observable $[S O(12)]_{O}$ is constructed as $12=\left(8_{S}, 1,1\right) \oplus(1,2,2)$, while in the second model the spinorials are constructed as $32=\left(8_{V}, 2,1\right) \oplus\left(8_{C}, 1,2\right)$ under the decomposition $S O(12) \rightarrow S O(8) \times S U(2) \times S U(2)$. At the core of the construction is the triality of the $S O(8)$ representations $8_{S} \leftrightarrow 8_{V} \leftrightarrow 8_{C}$. This property of the $S O(8)$ representations reproduces the standard decomposition of $S O(n+m) \rightarrow S O(n) \times S O(m)$ as $V^{n+m}=\left(V^{n}, 1\right) \oplus\left(1, V^{m}\right)$, and $S^{n+m}=\left(S^{n}, S^{m}\right) \oplus\left(C^{n}, C^{m}\right)$, for the vectorial and spinorial representations of $S O(n+m)$, respectively. The triality of the $S O(8)$ representations enables the twisted realisations of the GUT gauge group and representations, which is $S O(12)$ in the $N=2$ models and $S O(10)$ in $N=1$ models. This $S O(8)$ triality is the main property in the analysis of Section 3.

The transformation between the two models, (15) and (18), is induced by the discrete GGSO phase change

$$
\begin{aligned}
& c\left[\begin{array}{l}
b_{1} \\
z_{1}
\end{array}\right]=+1 \longrightarrow \\
& c\left[\begin{array}{l}
b_{1} \\
z_{1}
\end{array}\right]=-1 .
\end{aligned}
$$

In the models utilising the basis of (7), the map from sectors that produce vectorial representations of the observable $S O(12)$ group to sectors that produce spinorial representations is obtained by adding the basis vector $z_{0}$, which is similar to the $x$ map of $[18,19,40,41]$. The basis vector $z_{0}$ therefore acts as the spectral flow operator. It is a generator of the right-moving $N=2$ world-sheet supersymmetry in the models that preserve $(2,2)$ world-sheet supersymmetry. It is the mirror image of the basis vector $S$, which is the spectral flow operator on the fermionic side of the heterotic string. For appropriate choice of the discrete GGSO phases, 
either the vectorial states or the spinorial states are kept in the spectrum. The discrete phase modification in (19) induces the spinor-vector duality map in the $N=2$ model. The role of the basis vectors $z_{2}$ and $z_{3}$ in the models of (15) and (18) is to generate the twisted realisation of the gauge symmetry enhancement of the $S O(8)$ gauge groups arising from the null sector. We may further represent the spinor-vector duality in an orbifold representation [42] and translate the duality map in (19) to distinct choices of the toroidal background fields [24, 43]. Generalisation of the spectral map transformation between heterotic-string vacua was extended to Gepner models in [44].

\section{3. $D=2$ Model Classification}

In this section, we extend the classification of the symmetry groups to the case of compactifications to two dimensions. We develop the formalism and perform a complete classification in the simpler cases and partial classification in the more complex cases, where complexity here entails increasing number of basis vectors. The primary property which is exploited in our classification is the triality of the $S O(8)$ representations. In Section 4, we will employ an alternative method to construct the 24-dimensional Niemeier lattices. In Section 5, we will comment on the overlap and differences between our analysis in Sections 3 and 4 and that of Section 2.

We compactify the heterotic string to two dimensions. The two-dimensional free fermions in the light-cone gauge (in the usual notation $[28,29,31-39]$ ) are $\chi^{i}, y^{i}, \omega^{i}, i=$ $1, \ldots, 8$ (real left-moving fermions) and $\bar{y}^{i}, \bar{\omega}^{i}, i=1, \ldots, 8$ (real right-moving fermions), $\bar{\psi}^{A}, A=1, \ldots, 4, \bar{\eta}^{B}, B=$ $0,1,2,3, \bar{\phi}^{\alpha}, \alpha=1, \ldots, 8$ (complex right-moving fermions). The left- and right-moving real fermions are combined into complex fermions as $\rho_{i}=1 / \sqrt{2}\left(y_{i}+i \omega_{i}\right), i=1, \ldots, 8, \bar{\rho}_{i}=$ $1 / \sqrt{2}\left(\bar{y}_{i}+i \bar{\omega}_{i}\right), i=1, \ldots, 4, \bar{\rho}_{i}=1 / \sqrt{2}\left(\bar{y}_{i}+i \bar{\omega}_{i}\right), i=5, \ldots, 8$.

The class of models under investigation is generated by a maximal set $V$ of 7 basis vectors

$$
\begin{aligned}
& V=\left\{v_{1}, v_{2}, \ldots, v_{7}\right\}, \\
& v_{1}=\mathbf{1}=\left\{\chi^{1, \ldots, 8}, y^{1, \ldots, 8}, \omega^{1, \ldots, 8} \mid \bar{y}^{1, \ldots, 8}, \bar{\omega}^{1, \ldots, 8}, \bar{\eta}^{0,1,2,3},\right. \\
& \left.\bar{\psi}^{1, \ldots, 4}, \bar{\phi}^{1, \ldots, 8}\right\}, \\
& v_{2}=H_{L}=\left\{\chi^{1, \ldots, 8}, y^{1, \ldots, 8}, \omega^{1, \ldots, 8}\right\}, \\
& v_{3}=z_{1}=\left\{\bar{\phi}^{1, \ldots, 4}\right\}, \\
& v_{4}=z_{2}=\left\{\bar{\phi}^{5, \ldots, 8}\right\}, \\
& v_{5}=z_{3}=\left\{\bar{\psi}^{1, \ldots, 4}\right\}, \\
& v_{6}=z_{4}=\left\{\bar{\eta}^{0,1,2,3}\right\}, \\
& v_{7}=z_{5}=\left\{\bar{y}^{1, \ldots, 4}, \bar{\omega}^{1, \ldots, 4}\right\},
\end{aligned}
$$

with the corresponding matrix of one-loop GGSO projection coefficients

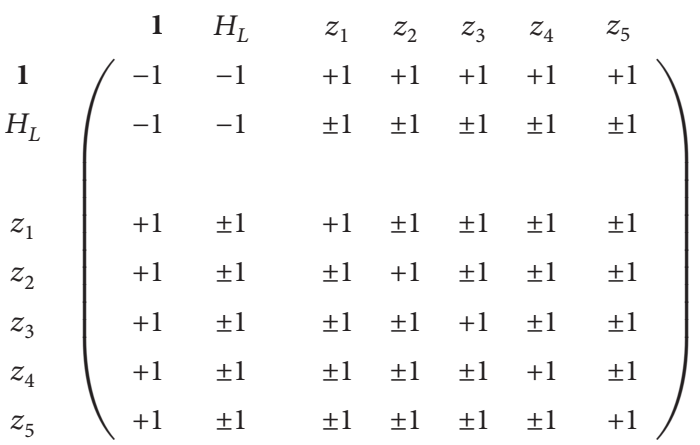

The analysis of the models is similar to the analysis in the four-dimensional case, where we define the GGSO projections in a similar way to (5), with the $\delta_{S}$ index being +1 in sectors in which the left-moving world-sheet fermions are antiperiodic and -1 in sectors in which they are periodic. With this definition of the GGSO projection, consistent with modular invariance, we can proceed to analyse the symmetry configurations.

3.1. Configurations. We analyse the various configurations that arise with an increasingly larger number of basis vectors. The simplest is the set $\left\{\mathbf{1}, H_{L}\right\}$. With this set, there is only one possible configuration with $S O(48)$ symmetry. Climbing the complexity ladder by adding the $z_{1}$ basis vector produces two possible configurations: $S O(8) \times S O(40)$ and $S O(48)$. The first is obtained from the untwisted vector states and the vector states from the sector $z_{1}$ are projected out, whereas the second is obtained by retaining the states from $z_{1}$ in the massless spectrum. The choice of the phase $c\left[\begin{array}{c}z_{1} \\ H_{L}\end{array}\right]= \pm 1$ selects between the two configurations. The next set is obtained by adding the basis vector $z_{2}$ yielding the set $\left\{\mathbf{1}, H_{L}, z_{1}, z_{2}\right\}$. The matrix of GGSO phases is given by

$$
\begin{aligned}
& 1 \begin{array}{llll}
H_{L} & z_{1} & z_{2}
\end{array} \\
& \begin{array}{c}
1 \\
H_{L} \\
z_{1} \\
z_{2}
\end{array} \quad\left(\begin{array}{cccc}
-1 & -1 & +1 & +1 \\
-1 & -1 & \pm 1 & \pm 1 \\
& & & \\
+1 & \pm 1 & +1 & \pm 1 \\
+1 & \pm 1 & \pm 1 & +1
\end{array}\right) \text {. }
\end{aligned}
$$

Only the phases above the diagonal are independent, whereas those on and below the diagonal are fixed by the modular invariance rules. Thus, in the configurations corresponding to (22), we have a total of three independent phases or eight possible configurations. Naturally, there are degeneracies in the space of configurations due to the permutation symmetries among $z_{i}$. With the basis corresponding to (22), we find a total of four independent configurations shown in Table 1.

We note that, with each subsequent basis set, the configurations of the smaller sets are reproduced. This is a recurring feature of string constructions $[18,19]$ and results from some generic $\theta$ function identities and redistribution of the vector 
TABLE 1: The configuration of the symmetry group with four basis vectors.

\begin{tabular}{lccc}
\hline$c\left[\begin{array}{l}z_{1} \\
H_{L}\end{array}\right]$ & $c\left[\begin{array}{l}z_{2} \\
H_{L}\end{array}\right]$ & $c\left[\begin{array}{l}z_{1} \\
z_{2}\end{array}\right]$ & Gauge group $G$ \\
\hline+ & + & + & $S O(16) \times S O(32)$ \\
+ & - & + & $S O(8) \times S O(40)$ \\
- & - & + & $S O(48)$ \\
- & - & - & $E_{8} \times S O(32)$ \\
\hline
\end{tabular}

states among the different sectors. We next add the additional basis vector $z_{3}$ producing a five-basis set $\left\{\mathbf{1}, H_{L}, z_{1}, z_{2}, z_{3}\right\}$. The phases matrix is given by

$$
\begin{gathered}
1 \\
H_{L} \\
z_{1} \\
z_{2} \\
z_{3}
\end{gathered} \quad\left(\begin{array}{ccccc}
-1 & -1 & +1 & +1 & +1 \\
-1 & -1 & \pm 1 & \pm 1 & \pm 1 \\
& & & & \\
+1 & \pm 1 & +1 & \pm 1 & \pm 1 \\
+1 & \pm 1 & \pm 1 & +1 & \pm 1 \\
+1 & \pm 1 & \pm 1 & \pm 1 & +1
\end{array}\right) .
$$

The untwisted symmetry is $\mathrm{SO}(8)_{1} \times \mathrm{SO}(8)_{2} \times \mathrm{SO}(8)_{3} \times$ $S O(24)$. In this case, there are a total of six independent phases producing 64 distinct possibilities. Out of those, we obtain seven distinct configurations shown in Table 2. Four of the resulting configurations are reproductions of previous cases and three are new. A complete analysis of all configurations has been performed in the case with five basis vectors.

The next step is to add an additional basis vector to the set. The set of basis vectors is then $\left\{\mathbf{1}, H_{L}, z_{1}, z_{2}, z_{3}, z_{4}\right\}$. The untwisted symmetry is $\mathrm{SO}(8)_{1} \times \mathrm{SO}(8)_{2} \times \mathrm{SO}(8)_{3} \times \mathrm{SO}(8)_{4} \times$ $S O(16)$. The sectors contributing to the symmetry group are the 0 sector and the 11 purely antiholomorphic sets:

$$
\begin{gathered}
G \\
=\left\{\begin{array}{c}
0, \\
z_{1}, z_{2}, z_{3}, z_{4}, \\
z_{1}+z_{2}, z_{1}+z_{3}, z_{1}+z_{4}, z_{2}+z_{3}, z_{2}+z_{4}, z_{3}+z_{4}, \\
\tilde{z}=\mathbf{1}+H_{L}+z_{1}+z_{2}+z_{3}+z_{4}
\end{array}\right\},
\end{gathered}
$$

where the 0 sector requires two oscillators acting on the vacuum in the gauge sector; the $z_{j}$ sectors require one oscillator; and $z_{i}+z_{j}$ and $\widetilde{z}$ require no oscillators. The matrix of GGSO phases is given by

$$
\begin{gathered}
1 \\
H_{L} \\
\\
z_{1} \\
z_{2} \\
z_{3} \\
z_{4}
\end{gathered} \quad\left(\begin{array}{cccccc}
-1 & -1 & +1 & +1 & +1 & +1 \\
-1 & -1 & \pm 1 & \pm 1 & \pm 1 & \pm 1 \\
& & & & & \\
+1 & \pm 1 & +1 & \pm 1 & \pm 1 & \pm 1 \\
+1 & \pm 1 & \pm 1 & +1 & \pm 1 & \pm 1 \\
+1 & \pm 1 & \pm 1 & \pm 1 & +1 & \pm 1 \\
+1 & \pm 1 & \pm 1 & \pm 1 & \pm 1 & +1
\end{array}\right) .
$$

There are 10 independent phases in (25) rendering a total of 1024 different possibilities with a complete analysis seemingly prohibitive. For a sample of the choices, we reproduce the previous seven configurations and obtain six new ones. The thirteen configurations are displayed in Table 3.

The fifth case in Table 3 is a new feature of the basis set corresponding to (25) as compared to the earlier cases. In all the previous cases, the symmetry was enhanced by one or more of the additional sectors, whereas in the case of the fifth row in Table 3 all enhancements are projected out. Thus, this set affords a larger set of projectors that facilitate projection of all enhancements. This is a recurring feature, which is frequently used in classification of fermionic string vacua in four dimensions. The last row in Table 3 corresponds to a model with $E_{8}^{3}$ symmetry, which is identified as one of the Niemeier lattices.

The next and final step is to add an additional basis vector which corresponds to the set given in (20) and the GGSO coefficients matrix in (21). The untwisted symmetry is $\mathrm{SO}(8)_{1} \times \mathrm{SO}(8)_{2} \times \mathrm{SO}(8)_{3} \times \mathrm{SO}(8)_{4} \times \mathrm{SO}(8)_{5} \times \mathrm{SO}(8)_{6}$, corresponding to the six sets of right-moving worldsheet complex fermions

$$
\begin{aligned}
& \left\{\left\{\bar{\rho}^{1,2,3,4}\right\} ;\left\{\bar{\rho}^{5,6,7,8}\right\} ;\left\{\bar{\psi}^{1,2,3,4}\right\} ;\left\{\bar{\eta}^{0,1,2,3}\right\} ;\left\{\bar{\phi}^{1,2,3,4}\right\} ;\right. \\
& \left.\left\{\bar{\phi}^{5,6,7,8}\right\}\right\} .
\end{aligned}
$$

The sectors contributing to the symmetry group are the 0 sector and the 21 purely antiholomorphic sets:

$$
G=\left\{\begin{array}{c}
0, \\
z_{1}, z_{2}, z_{3}, z_{4}, z_{5}, z_{6}, \\
z_{1}+z_{2}, z_{1}+z_{3}, z_{1}+z_{4}, z_{1}+z_{5}, z_{1}+z_{6}, \\
z_{2}+z_{3}, z_{2}+z_{4}, z_{2}+z_{5}, z_{2}+z_{6}, \\
z_{3}+z_{4}, z_{3}+z_{5}, z_{3}+z_{6}, \\
z_{4}+z_{5}, z_{4}+z_{6}, z_{5}+z_{6}
\end{array}\right\} \text {, }
$$

where $z_{6}=\mathbf{1}+H_{L}+z_{1}+z_{2}+z_{3}+z_{4}+z_{5}=$ $\left\{\bar{\phi}^{5,6,7,8}\right\}$. Similar to the previous cases, the 0 sector requires two oscillators acting on the vacuum in the right-moving sector to produce a massless state; the $z_{i}$ sectors require one oscillator; and $z_{i}+z_{j}$ with $i \neq j$ require no oscillators. All these cases require one oscillator acting on the vacuum in the left-moving sector. There are 15 independent phases in (21) rendering a total of 32768 possibilities, which requires a computerised analysis and is beyond our scope here.

All the sets that we introduced so far involve nonoverlapping periodic fermions; that is, the product between any two nontrivial basis vectors is $0 \bmod 4$. We can introduce additional basis vectors with two overlapping right-moving periodic fermions; that is, the product between the new basis vectors and two of those in (20) is 2. For example, a basis vector with $z_{7}=\left\{\bar{\rho}^{1,2}, \bar{\eta}^{2,3}\right\} \equiv 1$ has $z_{7} \cdot z_{1}=z_{7} \cdot z_{4}=2$. We can further envision breaking $H_{L}$ into three corresponding basis vectors $z_{0}, z_{8}$, and $z_{9}$ with $H_{L}=z_{0}+z_{8}+z_{9}$ and similarly 
TABLE 2: The configuration of the symmetry group with five basis vectors.

\begin{tabular}{lcccccc}
\hline$c\left[\begin{array}{l}z_{1} \\
H_{L}\end{array}\right]$ & $c\left[\begin{array}{l}z_{2} \\
H_{L}\end{array}\right]$ & $c\left[\begin{array}{l}z_{3} \\
H_{L}\end{array}\right]$ & $c\left[\begin{array}{l}z_{1} \\
z_{2}\end{array}\right]$ & $c\left[\begin{array}{l}z_{1} \\
z_{3}\end{array}\right]$ & $c\left[\begin{array}{l}z_{2} \\
z_{3}\end{array}\right]$ & Gauge group G \\
\hline+ & + & + & + & + & + & $S O(24) \times S O(24)$ \\
+ & + & + & + & + & - & $S O(8) \times S O(16) \times S O(24)$ \\
+ & + & - & + & + & + & $S O(16) \times S O(32)$ \\
- & - & + & + & + & + & $S O(8) \times S O(40)$ \\
- & - & + & - & + & + & $E_{8} \times S O(8) \times S O(24)$ \\
- & - & - & - & + & + & $S O(48)$ \\
- & - & - & - & + & $E_{8} \times S O(32)$ \\
\hline
\end{tabular}

TABLE 3: The configuration of the symmetry group with six basis vectors.

\begin{tabular}{ccccccccccc}
\hline$c\left[\begin{array}{l}z_{1} \\
H_{L}\end{array}\right]$ & $c\left[\begin{array}{l}z_{2} \\
H_{L}\end{array}\right]$ & $c\left[\begin{array}{l}z_{3} \\
H_{L}\end{array}\right]$ & $c\left[\begin{array}{l}z_{4} \\
H_{L}\end{array}\right]$ & $c\left[\begin{array}{l}z_{1} \\
z_{2}\end{array}\right]$ & $c\left[\begin{array}{l}z_{1} \\
z_{3}\end{array}\right]$ & $c\left[\begin{array}{l}z_{1} \\
z_{4}\end{array}\right]$ & $c\left[\begin{array}{l}z_{2} \\
z_{3}\end{array}\right]$ & $c\left[\begin{array}{l}z_{2} \\
z_{4}\end{array}\right]$ & $c\left[\begin{array}{l}z_{3} \\
z_{4}\end{array}\right]$ & Gauge group G \\
\hline+ & + & + & + & + & + & + & + & + & + & $E_{8} \times S O(32)$ \\
+ & + & + & + & + & + & + & + & + & - & $S O(16) \times S O(16) \times S O(16)$ \\
+ & + & + & + & + & + & + & + & - & - & $S O(16) \times S O(8) \times S O(8) \times S O(16)$ \\
+ & + & + & + & + & + & + & - & - & - & $E_{8} \times S O(24) \times S O(8)$ \\
+ & + & + & + & - & + & + & - & + & - & $S O(16) \times S O(8) \times S O(8) \times S O(8) \times S O(8)$ \\
+ & + & + & + & - & - & - & + & + & + & $S O(24) \times S O(16) \times S O(8)$ \\
+ & + & + & + & + & - & - & - & - & + & $E_{8} \times S O(16) \times S O(16)$ \\
- & + & + & + & + & + & + & + & + & + & $S O(24) \times S O(24)$ \\
+ & + & + & + & - & - & - & - & - & - & $S O(32) \times S O(16)$ \\
- & - & + & + & - & + & + & + & + & + & $E_{8} \times E_{8} \times S O(16)$ \\
- & - & - & - & + & + & + & + & + & + & $S O(48)$ \\
- & - & - & + & + & + & + & + & + & + & $S O(40) \times S O(8)$ \\
- & - & - & - & - & + & + & + & + & - & $E_{8} \times E_{8} \times E_{8}$ \\
\hline
\end{tabular}

introduce basis vectors with overlapping periodic complex fermions. A single mod 4 left-moving basis vector, with null assignment for the right-moving fermions, produces a Jacobi-like factor, $V_{8}-S_{8}$, in the partition function, which produces $N=4$ space-time supersymmetry in the fourdimensional models. Such nonoverlapping left-moving basis vectors produce a product of Jacobi-like identities, whereas basis vectors with overlapping periodic fermions break this identity in a familiar way from the four-dimensional models. The action of the basis vectors with overlapping periodic fermions is reminiscent of the orbifold action in Section 2 and combining a left-moving action with a right-moving one will entail precisely that. This will alter the sharp division between the left and the right movers and reduce the symmetry structures obtained with the 24-dimensional lattices. This looks similar to the case of toroidal orbifolds. Detailed analysis of these cases is beyond our scope here and will be reported in future work. What may be envisioned is that the symmetry structures of the four-dimensional models are rooted in the rich symmetry structures of the 24-dimensional lattices in two dimensions. In turn, the free fermionic construction may provide a set of simple tools that can be used to explore the properties of the 24-dimensional lattices. In the next section, we derive some of the Niemeier lattices by using the free fermionic tools.

\section{The Niemeier Lattices}

Some of the models we have already presented have the property that the modular invariant partition function factorises into a left- and a right-moving part:

$$
Z(\tau, \bar{\tau})=Z(\tau) Z(\bar{\tau})
$$

For models based on the set $\left\{H_{L}, H_{R}\right\}=\left\{\mathbf{1}, H_{R}\right\}$, which might also include some $z_{i}$ 's given in (20), this will happen if the phases between $H_{L}$ and any other vector are chosen appropriately $\left(c\left[\begin{array}{c}H_{L} \\ \text { anything }\end{array}\right]=-1\right)$.

Within the class of models with factorised partition functions, there is a subclass of models for which $Z(\tau)$ and $Z(\bar{\tau})$ are modular invariant by themselves. Particular cases of this type are models for which $Z(\tau)$ is a constant. These models display a Massive Spectrum Degeneracy Symmetry (MSDS) and have been studied in $[25,26]$. Here, we would like to focus more on the right-moving partition function, which for lattice compactifications is

$$
Z(\bar{\tau})=\frac{Z_{\Lambda}(\bar{\tau})}{\bar{\eta}(\bar{\tau})^{24}} .
$$

$\Lambda$ is the lattice on which the right-moving bosons are compactified. 
Since $Z(\bar{\tau})$ is modular invariant, $\Lambda$ must be an even, selfdual, 24-dimensional lattice (assuming a compactification to two dimensions). There are 24 such lattices classified by Niemeier [45] and they are presented in Table 4. With the exception of the Leech lattice that has no vectors of length 2, the vectors of length 2 of the remaining 23 lattices belong to the root lattices of simple Lie groups. However, knowing the components is not enough by itself to fully define a Niemeier lattice. One must also describe how conjugacy classes among different components are coupled with each other. This is given in terms of certain glue vectors. For example, the Niemeier lattice $D_{12}^{2}$ needs glue vectors $\{(s, v),(v, s)\}$, where $v$ and $s$ stand for the vector and the spinor conjugacy class of $D_{12}$. More details about this construction and a list of glue vectors for all the Niemeier lattices can be found in [46, 47].

Note that these lattices have been studied extensively in the past, especially in connection with moonshine. For example, the lattice $A_{1}^{24}$ carries a natural representation of the monster group $M_{24}$ and the Umbral Moonshine conjecture associates a finite group and a set of vector valued mock modular forms with each of these 23 Niemeier lattices (see [48] and the references therein).

Lattice compactifications have an equivalent fermionic description $[49,50]$. The main result of this section is Table 4 in which we give realisations of the Niemeier lattices in terms of free fermionic basis vectors. Note that even though many glue vectors need to be included for a description in the bosonic language, the free fermionic realisations of many of these lattices are quite succinct. This is a demonstration of the power of the free fermionic formalism for certain tasks and an example where the dictionary between bosons and fermions described in [49] can be used to provide new insights.

For example, let us look at the $D_{12}^{2}$ Niemeier lattice again. The straightforward way one might imagine implementing this in the free fermionic language is through the basis set $\left\{b_{1}, b_{2}, b_{3}, b_{4}\right\}$, where (remembering that the normalisation is twice the usual for weight vectors)

$$
\begin{aligned}
& b_{1}=\left\{1^{12}, 0^{12}\right\}, \\
& b_{2}=\left\{0^{12}, 1^{12}\right\}, \\
& b_{3}=\left\{1^{12}, 2,0^{11}\right\}, \\
& b_{4}=\left\{2,0^{11}, 1^{12}\right\} .
\end{aligned}
$$

$b_{1}$ and $b_{2}$ generate the two $D_{12}$ 's, whereas $b_{3}$ and $b_{4}$ are what one would naively write down to implement the glue vectors $(s, v)$ and $(v, s)$. On the other hand, the inclusion of $b_{3}$ and $b_{4}$ appears to be highly unconventional from a free fermionic model building perspective because they are not independent of $b_{1}$ and $b_{2}$ when considering mod 2 . The resolution to this paradox is to use the formula [49]

$$
c\left[\begin{array}{c}
\alpha+\delta \\
\alpha^{\prime}+\delta^{\prime}
\end{array}\right]=e^{(1 / 2) \pi i \delta \cdot \alpha^{\prime}} c\left[\begin{array}{c}
\alpha \\
\alpha^{\prime}
\end{array}\right]
$$

where $\delta$ and $\delta^{\prime}$ have only even entries and $\alpha, \alpha^{\prime}$ are arbitrary, to reduce $b_{3}$ and $b_{4}$ to $b_{1}$ and $b_{2}$, respectively. This also changes the phase $c\left[\begin{array}{l}b_{1} \\ b_{2}\end{array}\right]$ from 1 to -1 , hence verifying the corresponding entry in Table 4 . The same idea can be applied to fill in the rest of the table.

We tried to give realisations of the lattices using a small number of free fermionic basis vectors. However, for certain lattices, we were not able to do so and we had to use the following set of 12 basis vectors $\left\{g_{1}, g_{2}, \ldots, g_{12}\right\}$, known as the Golay generators:

$$
\left(\begin{array}{llllllllllllllllllllllll}
1 & 0 & 0 & 0 & 0 & 0 & 0 & 0 & 0 & 0 & 0 & 0 & 0 & 1 & 1 & 1 & 1 & 1 & 1 & 1 & 1 & 1 & 1 & 1 \\
0 & 1 & 0 & 0 & 0 & 0 & 0 & 0 & 0 & 0 & 0 & 0 & 1 & 1 & 1 & 0 & 1 & 1 & 1 & 0 & 0 & 0 & 1 & 0 \\
0 & 0 & 1 & 0 & 0 & 0 & 0 & 0 & 0 & 0 & 0 & 0 & 1 & 1 & 0 & 1 & 1 & 1 & 0 & 0 & 0 & 1 & 0 & 1 \\
0 & 0 & 0 & 1 & 0 & 0 & 0 & 0 & 0 & 0 & 0 & 0 & 1 & 0 & 1 & 1 & 1 & 0 & 0 & 0 & 1 & 0 & 1 & 1 \\
0 & 0 & 0 & 0 & 1 & 0 & 0 & 0 & 0 & 0 & 0 & 0 & 1 & 1 & 1 & 1 & 0 & 0 & 0 & 1 & 0 & 1 & 1 & 0 \\
0 & 0 & 0 & 0 & 0 & 1 & 0 & 0 & 0 & 0 & 0 & 0 & 1 & 1 & 1 & 0 & 0 & 0 & 1 & 0 & 1 & 1 & 0 & 1 \\
0 & 0 & 0 & 0 & 0 & 0 & 1 & 0 & 0 & 0 & 0 & 0 & 1 & 1 & 0 & 0 & 0 & 1 & 0 & 1 & 1 & 0 & 1 & 1 \\
0 & 0 & 0 & 0 & 0 & 0 & 0 & 1 & 0 & 0 & 0 & 0 & 1 & 0 & 0 & 0 & 1 & 0 & 1 & 1 & 0 & 1 & 1 & 1 \\
0 & 0 & 0 & 0 & 0 & 0 & 0 & 0 & 1 & 0 & 0 & 0 & 1 & 0 & 0 & 1 & 0 & 1 & 1 & 0 & 1 & 1 & 1 & 0 \\
0 & 0 & 0 & 0 & 0 & 0 & 0 & 0 & 0 & 1 & 0 & 0 & 1 & 0 & 1 & 0 & 1 & 1 & 0 & 1 & 1 & 1 & 0 & 0 \\
0 & 0 & 0 & 0 & 0 & 0 & 0 & 0 & 0 & 0 & 1 & 0 & 1 & 1 & 0 & 1 & 1 & 0 & 1 & 1 & 1 & 0 & 0 & 0 \\
0 & 0 & 0 & 0 & 0 & 0 & 0 & 0 & 0 & 0 & 0 & 1 & 1 & 0 & 1 & 1 & 0 & 1 & 1 & 1 & 0 & 0 & 0 & 1
\end{array}\right) .
$$

Note that we only show the right-moving components of the basis vectors here, with the understanding that the left-moving components are all zero. For a consistent free fermionic model, the vector $H_{L}$ (at least) should also be added 
TABLE 4: Free fermionic realizations of all inequivalent Niemeier lattices. Underline means permutations of all blocks separated by a comma. For example, the three basis vectors needed for $E_{8}^{3}$ are $\left\{1^{8}, 0^{8}, 0^{8}\right\},\left\{0^{8}, 1^{8}, 0^{8}\right\}$, and $\left\{0^{8}, 0^{8}, 1^{8}\right\}$. Only the 24 right-moving components of the basis vectors appear explicitly; the left-moving ones are understood to be zero. We also state explicitly which generalized phases in the upper triangular part of the phase matrix are not 1 . For a consistent free fermionic model, the vector $H_{L}$ (at least) should also be added to the set to ensure that vector $\mathbf{1}$ is generated.

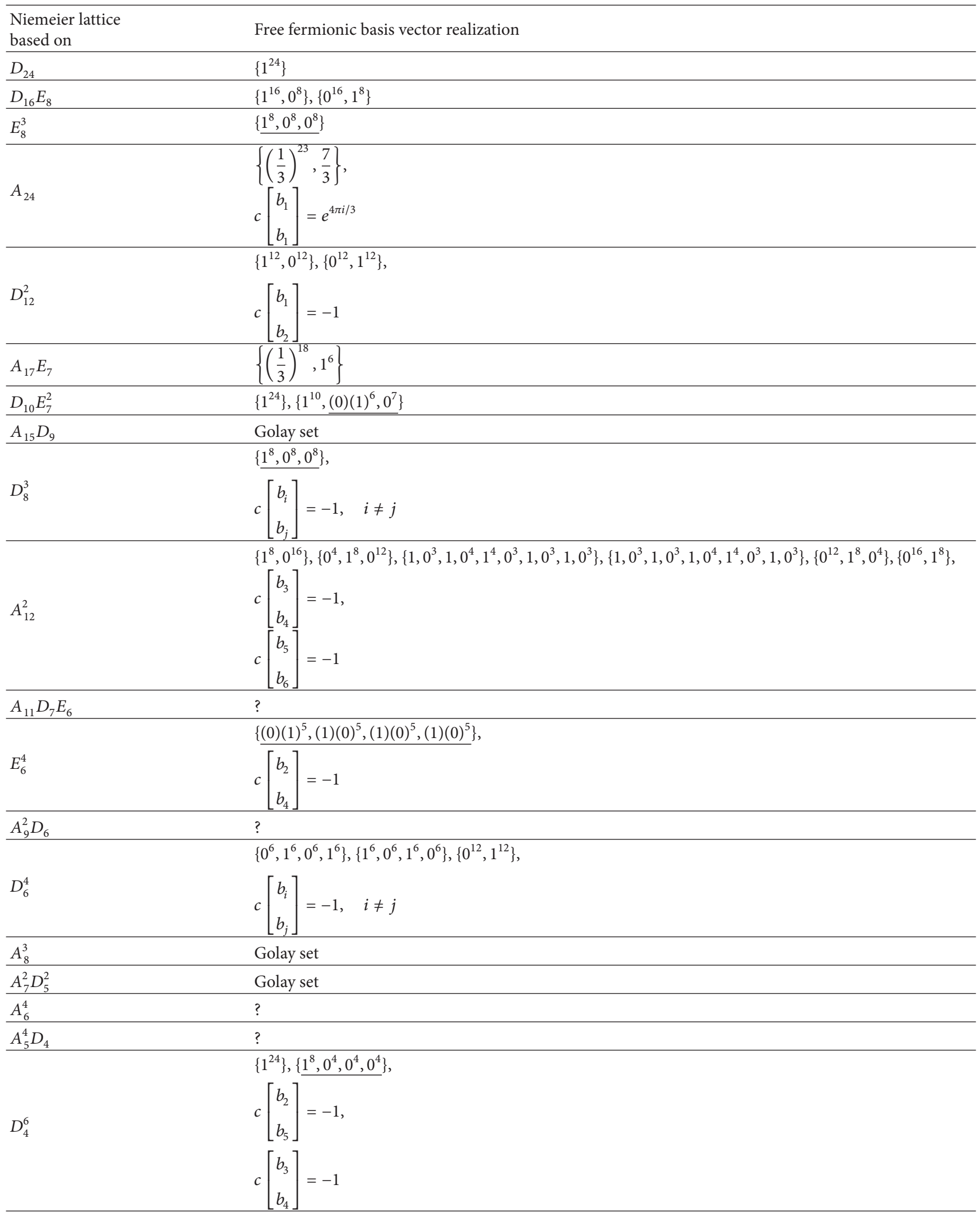


TABLE 4: Continued.

Niemeier lattice

based on

\begin{tabular}{ll}
\hline$A_{4}^{6}$ & Golay set \\
\hline$A_{3}^{8}$ & $?$ \\
\hline$A_{2}^{12}$ & $?$ \\
\hline$A_{1}^{24}$ & $?$ \\
\hline Leech & $?$ \\
\hline
\end{tabular}

to the set to ensure that vector $\mathbf{1}$ is generated. The defining phases $c\left[\begin{array}{l}g_{i} \\ g_{j}\end{array}\right]$ for the models that use this set are as follows:

$A_{8}^{3}: c$

$=\left(\begin{array}{cccccccccccc}-1 & -1 & -1 & -1 & -1 & -1 & -1 & -1 & -1 & -1 & -1 & -1 \\ * & 1 & 1 & 1 & -1 & -1 & 1 & 1 & -1 & -1 & 1 & 1 \\ * & * & 1 & 1 & -1 & -1 & 1 & 1 & -1 & -1 & 1 & 1 \\ * & * & * & 1 & -1 & -1 & 1 & 1 & -1 & -1 & 1 & 1 \\ * & * & * & * & -1 & -1 & 1 & 1 & -1 & -1 & 1 & 1 \\ * & * & * & * & * & -1 & 1 & 1 & -1 & -1 & 1 & 1 \\ * & * & * & * & * & * & 1 & 1 & -1 & -1 & 1 & 1 \\ * & * & * & * & * & * & * & 1 & -1 & -1 & 1 & 1 \\ * & * & * & * & * & * & * & * & -1 & -1 & 1 & 1 \\ * & * & * & * & * & * & * & * & * & -1 & 1 & 1 \\ * & * & * & * & * & * & * & * & * & * & 1 & 1 \\ * & * & * & * & * & * & * & * & * & * & * & 1\end{array}\right)$,

$A_{7}^{2} D_{5}^{2}: c$

$=\left(\begin{array}{cccccccccccc}-1 & -1 & -1 & -1 & -1 & -1 & -1 & -1 & -1 & -1 & -1 & -1 \\ * & 1 & 1 & 1 & -1 & -1 & -1 & -1 & 1 & 1 & -1 & -1 \\ * & * & 1 & 1 & -1 & -1 & -1 & -1 & 1 & 1 & -1 & -1 \\ * & * & * & 1 & -1 & -1 & -1 & -1 & 1 & 1 & -1 & -1 \\ * & * & * & * & -1 & -1 & -1 & -1 & 1 & 1 & -1 & -1 \\ * & * & * & * & * & -1 & -1 & -1 & 1 & 1 & -1 & -1 \\ * & * & * & * & * & * & -1 & -1 & 1 & 1 & -1 & -1 \\ * & * & * & * & * & * & * & -1 & 1 & 1 & -1 & -1 \\ * & * & * & * & * & * & * & * & 1 & 1 & -1 & -1 \\ * & * & * & * & * & * & * & * & * & 1 & -1 & -1 \\ * & * & * & * & * & * & * & * & * & * & -1 & -1 \\ * & * & * & * & * & * & * & * & * & * & * & -1\end{array}\right)$,

$A_{15} D_{9}: c$

$$
=\left(\begin{array}{cccccccccccc}
-1 & -1 & -1 & -1 & -1 & -1 & -1 & -1 & -1 & -1 & -1 & -1 \\
* & 1 & 1 & 1 & 1 & 1 & 1 & 1 & 1 & 1 & -1 & -1 \\
* & * & 1 & 1 & 1 & 1 & 1 & 1 & 1 & 1 & -1 & -1 \\
* & * & * & 1 & 1 & 1 & 1 & 1 & 1 & 1 & -1 & -1 \\
* & * & * & * & 1 & 1 & 1 & 1 & 1 & 1 & -1 & -1 \\
* & * & * & * & * & 1 & 1 & 1 & 1 & 1 & -1 & -1 \\
* & * & * & * & * & * & 1 & 1 & 1 & 1 & -1 & -1 \\
* & * & * & * & * & * & * & 1 & 1 & 1 & -1 & -1 \\
* & * & * & * & * & * & * & * & 1 & 1 & -1 & -1 \\
* & * & * & * & * & * & * & * & * & 1 & -1 & -1 \\
* & * & * & * & * & * & * & * & * & * & -1 & -1 \\
* & * & * & * & * & * & * & * & * & * & * & -1
\end{array}\right),
$$

$A_{4}^{6}: c$

$$
=\left(\begin{array}{cccccccccccc}
-1 & -1 & -1 & -1 & -1 & -1 & -1 & -1 & -1 & -1 & -1 & -1 \\
* & 1 & 1 & 1 & 1 & 1 & 1 & 1 & 1 & 1 & 1 & 1 \\
* & * & 1 & 1 & 1 & 1 & 1 & 1 & 1 & 1 & 1 & 1 \\
* & * & * & -1 & -1 & -1 & -1 & -1 & -1 & -1 & -1 & -1 \\
* & * & * & * & -1 & -1 & -1 & -1 & -1 & -1 & -1 & -1 \\
* & * & * & * & * & 1 & 1 & 1 & 1 & 1 & 1 & 1 \\
* & * & * & * & * & * & 1 & 1 & 1 & 1 & 1 & 1 \\
* & * & * & * & * & * & * & -1 & -1 & -1 & -1 & -1 \\
* & * & * & * & * & * & * & * & -1 & -1 & -1 & -1 \\
* & * & * & * & * & * & * & * & * & 1 & 1 & 1 \\
* & * & * & * & * & * & * & * & * & * & 1 & 1 \\
* & * & * & * & * & * & * & * & * & * & * & 1
\end{array}\right) .
$$

Note that there are some entries in Table 4 for which we were unable to provide concrete realisations. We conjecture that these cases can also be given in terms of the Golay basis vectors for certain phases. However, the fact that there are $2^{78}$ a priori different phases that are allowed by modular invariance makes it computationally difficult to verify (or disprove) this claim.

For all the models presented in Table 4, an independent check of correctness can be performed by calculating the partition function (restricted to the right-moving sector) of the proposed free fermionic realisation via the formula

$$
Z(\bar{\tau})=\sum_{\text {sectors } \alpha, \beta} c\left[\begin{array}{l}
\alpha \\
\beta
\end{array}\right] \bar{\theta}\left[\begin{array}{l}
\alpha \\
\beta
\end{array}\right]
$$

and we check that it matches the partition function of the Niemeier lattices given as [51]

$$
Z(\bar{\tau})=J(\bar{\tau})+24(h+1),
$$

where $h$ is the corresponding Coxeter number (given, e.g., in $[46])$ and $J(\bar{\tau})$ is the unique modular invariant with zero constant term (i.e., $J(\bar{\tau})=j(\bar{\tau})-744$ ). Note in particular that the partition functions of all these lattices (and those of the corresponding free fermionic models) only differ in their constant term. The massive spectra of all the models are identical.

The fact that the modular invariant phases in the partition function can be adjusted to couple conjugacy classes among 
different factors is quite surprising and, to our knowledge, has not been noticed before. It demonstrates an interesting interplay between gluing lattices and modularity and allows for a deeper understanding of the spinor-vector duality: for lattice compactifications, adjusting the generalised phases couples different conjugacy classes among different factors leading to different lattices. In a similar way, for orbifold compactifications, adjusting certain generalised phases leads to spinor-vector dual models.

\section{Conclusions}

The heterotic-string models in the free fermionic formulation are among the most realistic string models constructed to date [31-39]. They correspond to $Z_{2} \times Z_{2}$ toroidal orbifold constructions at special points in the moduli space $[49,52-$ 57]. Their phenomenological properties raise the possibility that the true string vacuum shares some of their underlying properties. It is therefore of immense interest to explore what those underlying properties are. It is of course also plausible that the true string vacuum does not belong to this class, and for that purpose other classes of interesting string vacua (see, e.g., [58-66]) should be investigated and their underlying properties explored.

A particular subclass of free fermionic models are those that allow for a light extra $Z^{\prime}$, with its distinct low scale signature via diphoton excess. The construction of the string model utilised the spinor-vector duality, which is akin to mirror symmetry $[67,68]$. A realisation of the spinor-vector duality relies on the triality of the $S O(8)$ representations. In particular, this triality enables a large range of possibilities for the GGSO phases to produce the same symmetry groups. The $S O(8)$ triality property is also at the core of the well known Jacobi identity and the ensuing space-time supersymmetry.

In this paper, we explored the symmetry structures of heterotic-string vacua compactified to two dimensions. Our primary motivation is to seek the origin of the fourdimensional spinor-vector duality in the symmetry structure of 24-dimensional lattices that are obtained in the two-dimensional compactifications. This is analogous to the MSDS symmetry which is similarly rooted in twodimensional compactifications. We discussed in Section 2 how the spinor-vector duality is rooted in the triality property of $S O(8)$ representations and we used this triality property in Section 3 to classify some of the symmetries of the two-dimensional compactifications. Self-duality under the spinor-vector duality played a key role in the construction of the $Z^{\prime}$ model of [5], with its distinctive signature via diphoton excess $[4,6]$. Thus, a basic property underlying the string vacua is tied to a phenomenological model with its distinct experimental signature. In Section 4, we derived a representation of some of the Niemeier lattices in the free fermionic formulation. The properties of 24-dimensional lattices and their moonshine symmetries are of growing interest in the literature [69-76]. In this context, it is not implausible that the free fermionic methods can add to the set of tools that can be used to explore the underlying mathematical structures. How, and whether, these mathematical phenomena manifest themselves in physical observable is the arena we will explore in future publications.

\section{Conflicts of Interest}

The authors declare that there are no conflicts of interest regarding the publication of this paper.

\section{Acknowledgments}

Alon E. Faraggi would like to thank the Simons Center for Geometry and Physics and the Oxford Particle Theory Group for their hospitality. This work is supported in part by STFC Consolidated Grant ST/L000431/1.

\section{References}

[1] ATLAS Collaboration, G. Aad et al., ATLAS-CONF-2015-081.

[2] CMS Collaboration, S. Chatrchyan et al., CMS PAS EXO15-004.

[3] A. Strumia, "Interpreting the $750 \mathrm{GeV}$ digamma excess: a review," Tech. Rep. CERN-TH-2016-131, 2016, https://arxiv .org/abs/1605.09401.

[4] A. E. Faraggi and J. Rizos, "The $750 \mathrm{GeV}$ di-photon LHC excess and extra $Z^{\prime} \mathrm{s}$ in heterotic-string derived models," European Physical Journal C, vol. 76, p. 170, 2016.

[5] A. E. Faraggi and J. Rizos, "A light $Z$ ' heterotic-string derived model," Nuclear Physics B, vol. 895, pp. 233-247, 2015.

[6] J. Ashfaque, L. Delle Rose, A. E. Faraggi, and C. Marzo, "The LHC di-photon excess and gauge coupling unification in extra $Z^{\prime}$ heterotic-string derived models," European Physical Journal C, vol. 76, no. 10, article 570, 26 pages, 2016.

[7] ATLAS Collaboration, G. Aad et al, ATLAS-CONF-2016-059.

[8] CMS Collaboration, S. Chatrchyan et al, CMS-PAS-EXO-16027.

[9] P. Langacker, “The physics of heavy $Z$ ' gauge bosons," Reviews of Modern Physics, vol. 81, p. 1199, 2009.

[10] A. Leike, "The phenomenology of extra neutral gauge bosons," Physics Reports, vol. 317, pp. 143-250, 1999.

[11] J. L. Hewett and T. G. Rizzo, "Low-energy phenomenology of superstring-inspired E6 models," Physics Reports, vol. 183, no. 5-6, pp. 193-381, 1989.

[12] A. E. Faraggi, "Proton stability and superstring $Z^{\prime}$ ", Physics Letters, Section B: Nuclear, Elementary Particle and HighEnergy Physics, vol. 499, no. 1-2, pp. 147-157, 2001.

[13] A. E. Faraggi and V. M. Mehta, "Proton stability and light $Z^{\prime}$ inspired by string derived models," Physical Review D Particles, Fields, Gravitation and Cosmology, vol. 84, no. 8, Article ID 086006, 2011.

[14] A. E. Faraggi and V. M. Mehta, "Proton stability, gauge coupling unification, and a light $Z^{\prime}$ in heterotic-string models," Physical Review D - Particles, Fields, Gravitation and Cosmology, vol. 88, no. 2, Article ID 025006, 2013.

[15] P. Athanasopoulos, A. E. Faraggi, and V. M. Mehta, "Light $Z$ ' in heterotic string standardlike models," Physical Review D Particles, Fields, Gravitation and Cosmology, vol. 89, no. 10, Article ID 105023, 2014. 
[16] A. Gregori, C. Kounnas, and J. Rizos, "Classification of the $N=2, Z_{2} \times Z_{2}$-symmetric type II orbifolds and their type II asymmetric duals," Nuclear Physics B, vol. 549, no. 1-2, pp. 1662, 1999.

[17] A. E. Faraggi, C. Kounnas, S. E. M. Nooij, and J. Rizos, "Classification of the chiral $Z_{2} \times Z_{2}$ fermionic models in the heterotic superstring," Nuclear Physics B, vol. 695, no. 1-2, pp. 41-72, 2004.

[18] A. E. Faraggi, C. Kounnas, and J. Rizos, "Chiral family classification of fermionic $Z_{2} \times Z_{2}$ heterotic orbifold models," Physics Letters B, vol. 648, no. 1, pp. 84-89, 2007.

[19] A. E. Faraggi, C. Kounnas, and J. Rizos, "Spinor-vector duality in fermionic $Z_{2} \times Z_{2}$ heterotic orbifold models," Nuclear Physics B, vol. 774, no. 1-3, pp. 208-231, 2007.

[20] B. Assel, K. Christodoulides, A. E. Faraggi, C. Kounnas, and J. Rizos, "Exophobic quasi-realistic heterotic string vacua," Physics Letters B, vol. 683, no. 4-5, pp. 306-313, 2010.

[21] B. Assel, K. Christodoulides, A. E. Faraggi, C. Kounnas, and J. Rizos, "Classification of heterotic Pati-Salam models," Nuclear Physics B, vol. 844, no. 3, pp. 365-396, 2011.

[22] A. E. Faraggi, J. Rizos, and H. Sonmez, "Classification of flipped $S U(5)$ heterotic-string vacua," Nuclear Physics B, vol. 886, pp. 202-242, 2014.

[23] H. Sonmez, "Flipped SU(5) breaking basis vector," Physical Review D, vol. 93, no. 12, 125002, 11 pages, 2016.

[24] A. E. Faraggi, I. Florakis, T. Mohaupt, and M. Tsulaia, "Conformal aspects of spinor-vector duality," Nuclear Physics B, vol. 848, no. 2, pp. 332-371, 2011.

[25] C. Kounnas, "Massive boson-fermion degeneracy and the early structure of the universe," Fortschritte der Physik. Progress of Physics, vol. 56, no. 11-12, pp. 1143-1156, 2008.

[26] I. Florakis and C. Kounnas, "Orbifold symmetry reductions of massive boson-fermion degeneracy," Nuclear Physics B, vol. 820, no. 1-2, pp. 237-268, 2009.

[27] A. E. Faraggi, C. Kounnas, and J. Rizos, "Spinor-vector duality in $N=2$ heterotic string vacua," Nuclear Physics B, vol. 799, no. 1-2, pp. 19-33, 2008.

[28] I. Antoniadis, C. P. Bachas, and C. Kounnas, "Four-dimensional superstrings," Nuclear Physics. B. Theoretical, Phenomenological, and Experimental High Energy Physics. Quantum Field Theory and Statistical Systems, vol. 289, no. 1, pp. 87-108, 1987.

[29] H. Kawai, D. C. Lewellen, and S. H. Tye, "Construction of fermionic string models in four dimensions," Nuclear Physics. B. Theoretical, Phenomenological, and Experimental High Energy Physics. Quantum Field Theory and Statistical Systems, vol. 288, no. 1, pp. 1-76, 1987.

[30] H. Kawai, D. C. Lewellen, and S.-H. H. Tye, "Classification of closed-fermionic-string models," Physical Review D, vol. 34, no. 12, pp. 3794-3804, 1986.

[31] I. Antoniadis, J. Ellis, J. S. Hagelin, and D. V. Nanopoulos, "The flipped SU(5) $\times$ U(1) string model revamped," Physics Letters B, vol. 231, no. 1-2, pp. 65-74, 1989.

[32] A. E. Faraggi, D. V. Nanopoulos, and K. Yuan, "A standard-like model in the four-dimensional free fermionic string formulation," Nuclear Physics, Section B, vol. 335, no. 2, pp. 347-362, 1990.

[33] I. Antoniadis, G. K. Leontaris, and J. Rizos, "A three-generation SU(4) $\times \mathrm{O}(4)$ string model," Physics Letters B, vol. 245, no. 2, pp. 161-168, 1990.

[34] A. E. Faraggi, "A new standard-like model in the four dimensional free fermionic string formulation," Physics Letters B, vol. 278, no. 1-2, pp. 131-139, 1992.
[35] A. E. Faraggi, "Construction of realistic standard-like models in the free fermionic superstring formulation," Nuclear Physics. B. Theoretical, Phenomenological, and Experimental High Energy Physics. Quantum Field Theory and Statistical Systems, vol. 387, no. 2, pp. 239-262, 1992.

[36] G. B. Cleaver, A. E. Faraggi, and D. V. Nanopoulos, "String derived MSSM and M-theory unification," Physics Letters. B. Particle Physics, Nuclear Physics and Cosmology, vol. 455, no. 1-4, pp. 135-146, 1999.

[37] G. K. Leontaris and J. Rizos, " $N=2$ supersymmetric $S U(4) \times$ $S U(2)_{L} \times S U(2)_{R}$ effective theory from the weakly coupled heterotic superstring," Nuclear Physics B, vol. 554, no. 1-2, pp. 3-49, 1999.

[38] G. B. Cleaver, A. E. Faraggi, and C. Savage, "Left-right symmetric heterotic-string derived models," Physical Review. D. Third Series, vol. 63, no. 6, Article ID 066001, 2001.

[39] A. E. Faraggi, E. Manno, and C. Timirgaziu, "Minimal standard heterotic string models," European Physical Journal C, vol. 50, no. 3, pp. 701-710, 2007.

[40] A. E. Faraggi, "Generation mass hierarchy in superstring derived models," Nuclear Physics, Section B, vol. 407, no. 1, pp. 57-72, 1993.

[41] A. E. Faraggi, "Partition functions of NAHE-based free fermionic string models," Physics Letters, Section B: Nuclear, Elementary Particle and High-Energy Physics, vol. 544, no. 1-2, pp. 207-214, 2002.

[42] C. Angelantonj, A. E. Faraggi, and M. Tsulaia, "Spinor-vector duality in heterotic string orbifolds," Journal of High Energy Physics, vol. 1007, no. 7, article no. 4, 2010.

[43] T. Catelin-Jullien, A. E. Faraggi, C. Kounnas, and J. Rizos, "Spinor-vector duality in heterotic SUSY vacua," Nuclear Physics. B. Theoretical, Phenomenological, and Experimental High Energy Physics. Quantum Field Theory and Statistical Systems, vol. 812, no. 1-2, pp. 103-127, 2009.

[44] P. Athanasopoulos, A. E. Faraggi, and D. Gepner, "Spectral flow as a map between $N=(2,0)$-models," Physics Letters B, vol. 735, pp. 357-363, 2014.

[45] H.-V. Niemeier, "Definite quadratische formen der dimension 24 und diskriminante 1," Journal of Number Theory, vol. 5, pp. 142-178, 1973.

[46] J. H. Conway and N. J. A. Sloane, Sphere Packing, Lattices and Groups, Springer, New York, NY, USA, 1998.

[47] W. Ebeling, "Lattices and Codes: A Course Partially Based on Lectures by Friedrich Hirzebruch," in Advanced Lectures in Mathematics, Springer, New York, NY, USA, 2012.

[48] M. C. N. Cheng, J. F. R. Duncan, and J. A. Harvey, "Umbral moonshine and the niemeier lattices," Research in the Mathematical Sciences, vol. 1, no. 3, 2014.

[49] P. Athanasopoulos, A. E. Faraggi, S. G. Nibbelink, and V. M. Mehta, "Heterotic free fermionic and symmetric toroidal orbifold models," Journal of High Energy Physics, vol. 2016, no. 4, article no. 38, 2016.

[50] P. Athanasopoulos, Relations in the space of $(2,0)$ heterotic string models [Ph.D. thesis], University of Liverpool, Liverpool, England, 2016.

[51] L. Dixon, P. Ginsparg, and J. Harvey, "Beauty and the beast: superconformal symmetry in a Monster module," Communications in Mathematical Physics, vol. 119, no. 2, pp. 221-241, 1988.

[52] A. E. Faraggi, " $Z_{2} \times Z_{2}$ orbifold compactification as the origin of realistic free fermionic models," Physics Letters B, vol. 326, no. 1-2, pp. 62-68, 1994. 
[53] E. Kiritsis and C. Kounnas, "Perturbative and non-perturbative partial supersymmetry breaking: $N=4 \rightarrow N=2 \rightarrow N=1$," Nuclear Physics B, vol. 503, no. 1-2, pp. 117-156, 1997.

[54] P. Berglund, J. Ellis, A. E. Faraggi, D. V. Nanopoulos, and Z. Qiu, "Toward the $M(F)$-theory embedding of realistic free-fermion models," Physics Letters B, vol. 433, no. 3-4, pp. 269-278, 1998.

[55] P. Berglund, J. Ellis, A. E. Faraggi, D. V. Nanopoulos, and Z. Qiu, "On elevating free-fermion $z_{2} \times z_{2}$ orbifolds models to compactifications of $F$ theory," International Journal of Modern Physics A, vol. 15, no. 9, p. 1345, 2000.

[56] R. Donagi and A. E. Faraggi, "On the number of chiral generations in $Z_{2} \times Z_{2}$ orbifolds," Nuclear Physics B, vol. 694, no. 1-2, pp. 187-205, 2004.

[57] A. E. Faraggi, S. Förste, and C. Timirgaziu, " $z_{2} \times z_{2}$ heterotic orbifold models of non factorisable six dimensional toroidal manifolds," Journal of High Energy Physics, vol. 608, no. 8, article no. 057, 2006.

[58] L. Ibanez, J. E. Kim, H. P. Nilles, and F. Quevedo, "Orbifold compactifications with three families of $\mathrm{Su}(3) \times \mathrm{Su}(2) \times \mathrm{U}(1)^{n}$," Physics Letters B, vol. 191, no. 3, pp. 282-286, 1987.

[59] K. R. Dienes, "New string partition functions with vanishing cosmological constant," Physical Review Letters, vol. 65, no. 16, pp. 1979-1982, 1990.

[60] K. R. Dienes, "Statistics on the heterotic landscape: gauge groups and cosmological constants of four-dimensional heterotic strings," Physical Review. D. Third Series, vol. 73, no. 10, 106010, 33 pages, 2006.

[61] R. Blumenhagen, F. Gmeiner, G. Honecker, D. Lüst, and T. Weigand, "The statistics of supersymmetric D-brane models," Nuclear Physics B, vol. 713, no. 1-3, pp. 83-135, 2005.

[62] F. Denef and M. R. Douglas, "Distributions of flux vacua," Journal of High Energy Physics, vol. 405, article 72, 2004.

[63] T. Kobayashi, S. Raby, and R.-J. Zhang, "Searching for realistic $4 \mathrm{~d}$ string models with a Pati-Salam symmetry. Orbifold grand unified theories from heterotic string compactification on a $\mathrm{Z}_{6}$ orbifold," Nuclear Physics B, vol. 704, no. 1-2, pp. 3-55, 2005.

[64] B. S. Acharya, F. Denef, and R. Valadro, "Statistics of $M$ theory vacua," The Journal of High Energy Physics, vol. 506, article 56, 2005.

[65] M. R. Douglas and W. Taylor, "The landscape of intersecting brane models," Journal of High Energy Physics, vol. 701, article 31, 2007.

[66] O. Lebedev, H. P. Nilles, S. Raby, S. Ramos-Sánchez, M. Ratz, P. K. S. Vaudrevange et al., "A mini-landscape of exact MSSM spectra in heterotic orbifolds," Physics Letters B, vol. 645, no. 1, pp. 88-94, 2007.

[67] B. R. Greene and M. R. Plesser, "Duality in Calabi-Yau moduli space," Nuclear Physics, Section B, vol. 338, no. 1, pp. 15-37, 1990.

[68] C. Vafa and E. Witten, "On orbifolds with discrete torsion," Journal of Geometry and Physics, vol. 15, no. 3, pp. 189-214, 1995.

[69] T. Eguchi, "Professor nambu, string theory and moonshine phenomenon," Journal of High Energy Physics, 8 pages, 2016, https://arxiv.org/abs/1608.06036.

[70] M. C. N. Cheng, P. de Lange, and D. P. Z. Whalen, "Generalised umbral moonshine," Journal of High Energy Physics, 45 pages, 2016, https://arxiv.org/abs/1608.07835.

[71] V. V. Nikulin, "Degenerations of Kahlerian K3 surfaces with finite symplectic automorphism groups. III," 2016, https://arxiv.org/abs/1608.04373.
[72] S. Kachru, "Elementary introduction to Moonshine," 2016, https://arxiv.org/abs/1605.00697.

[73] S. M. Harrison, "Extremal chiral $N=4$ SCFT with $c=24$," Journal of High Energy Physics, vol. 2016, no. 11, article 6, 2016.

[74] N. M. Paquette, D. Persson, and R. Volpato, "Monstrous BPSalgebras and the superstring origin of moonshine," Journal of High Energy Physics, 2016, https://arxiv.org/abs/1601.05412.

[75] A. Taormina and K. Wendland, "The overarching finite symmetry group of Kummer surfaces in the Mathieu group M 24," Journal of High Energy Physics, vol. 308, no. 8, article no. 125, 2013.

[76] M. R. Gaberdiel, S. Hohenegger, and R. Volpato, "Mathieu twining characters for K3," Journal of High Energy Physics, vol. 1010 , no. 9, article no. 62,2010 


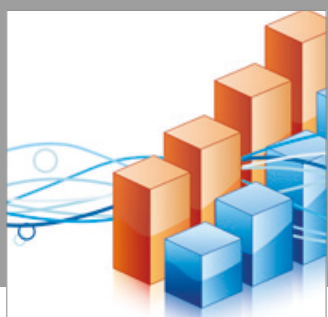

Advances in

Operations Research

vatersals

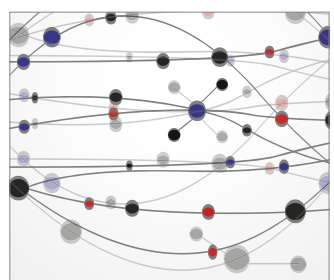

\section{The Scientific} World Journal
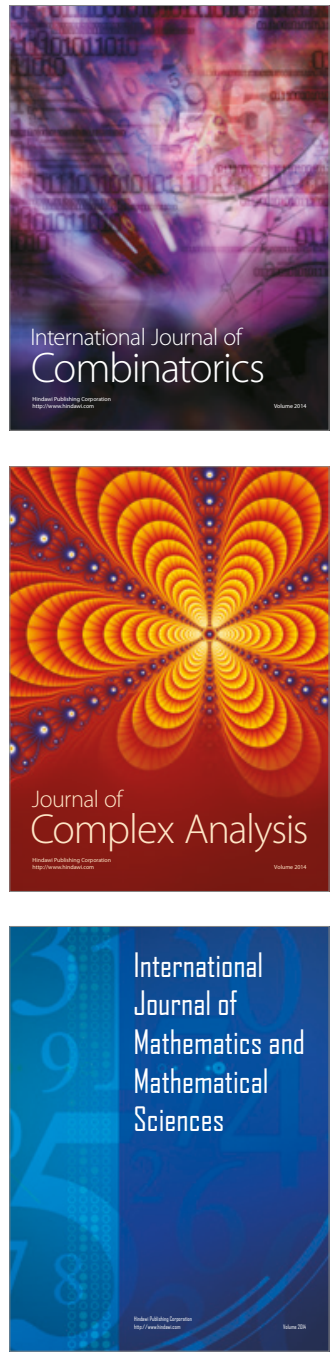
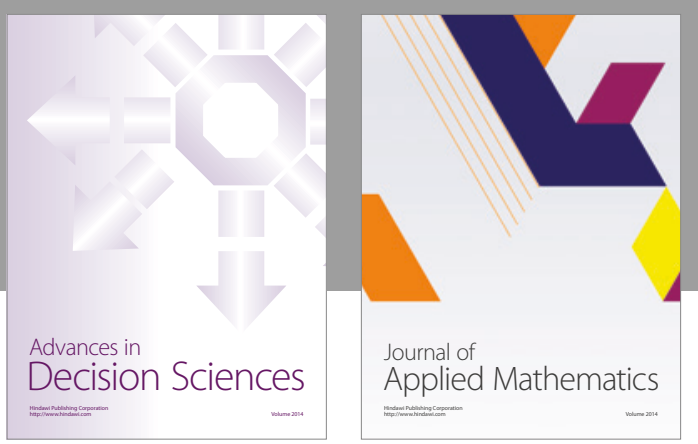

Algebra

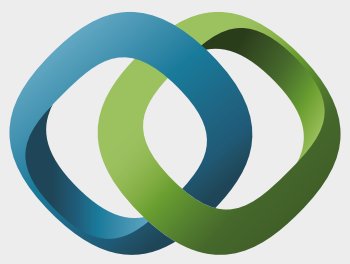

\section{Hindawi}

Submit your manuscripts at

https://www.hindawi.com
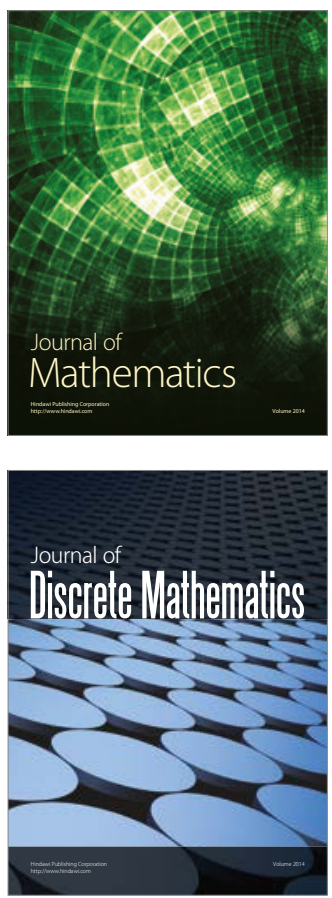

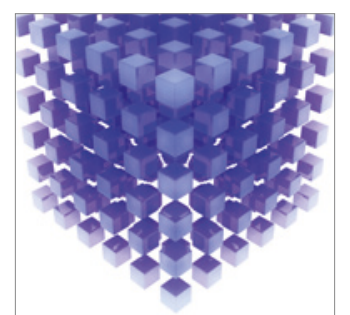

Mathematical Problems in Engineering
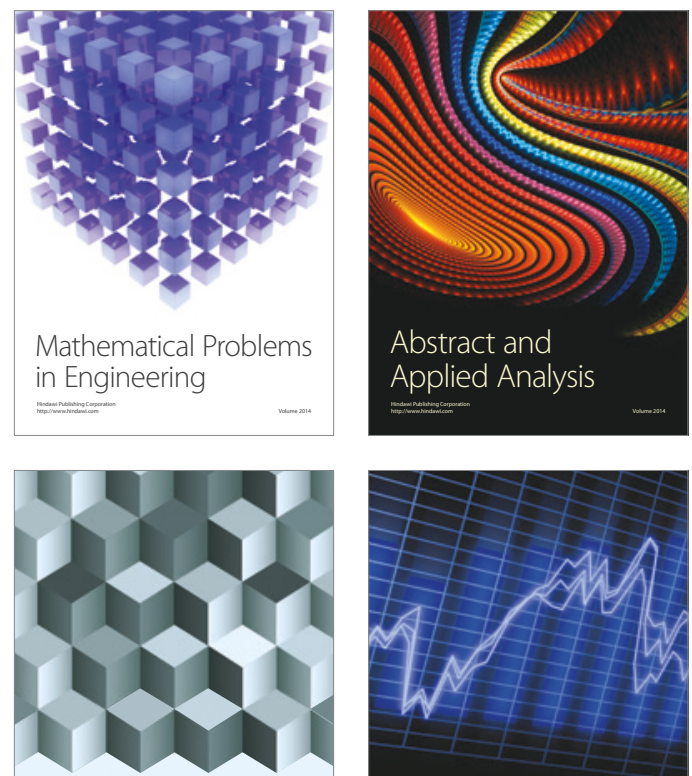

Journal of

Function Spaces

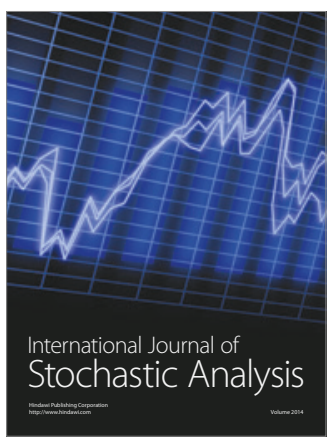

Probability and Statistics
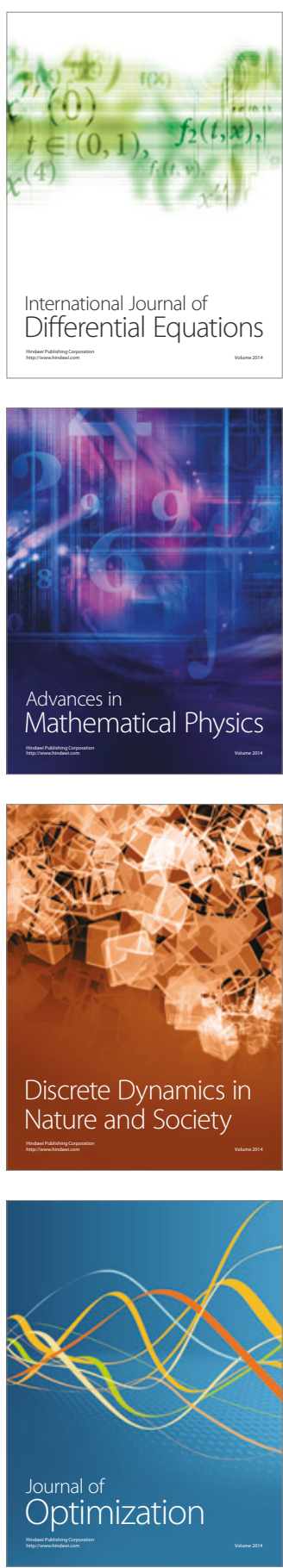